\title{
Application of Parameterized Hesitant Fuzzy Soft Set Theory in Decision Making
}

\author{
Zahari Md Rodzi1,2,*, Abd Ghafur Ahmad $^{1}$ \\ ${ }^{1}$ School of Mathematical Science, Faculty of Science and Technology, Universiti Kebangsaan Malaysia, Malaysia \\ ${ }^{2}$ Faculty of Computer and Mathematical Science, Universiti Teknologi MARA Campus Seremban, Malaysia
}

Received December 24, 2019; Revised March 12, 2020; Accepted April 27, 2020

Copyright $\odot 2020$ by authors, all rights reserved. Authors agree that this article remains permanently open access under the terms of the Creative Commons Attribution License 4.0 International License

\begin{abstract}
In this paper, by combining hesitant fuzzy soft sets (HFSSs) and fuzzy parameterized, we introduce the idea of a new hybrid model, fuzzy parameterized hesitant fuzzy soft sets (FPHFSSs). The benefit of this theory is that the degree of importance of parameters is being provided to HFSSs directly from decision makers. In addition, all the information is represented in a single set in the decision making process. Then, we likewise ponder its basic operations such as AND, OR, complement, union and intersection. The basic properties such as associative, distributive and de Morgan's law of FPHFSSs are proven. Next, in order to resolve the multi-criteria decision making problem (MCDM), we present arithmetic mean score and geometry mean score incorporated with hesitant degree of FPHFSSs in TOPSIS. This algorithm can cater some existing approach that suggested to add such elements to a shorter hesitant fuzzy element, rendering it equivalent to another hesitant fuzzy element, or to duplicate its elements to obtain two sequence of the same length. Such approaches would break the original data structure and modify the data. Finally, to demonstrate the efficacy and viability of our process, we equate our algorithm with existing methods.
\end{abstract}

Keywords Fuzzy Soft Set, Fuzzy Hesitant, Fuzzy Parameterized Hesitant Fuzzy Soft Sets

\section{Introduction}

The concept of fuzzy sets presented by Zadeh [1] has conquered an enormous achievement in numerous fields. The extension of the fuzzy sets and one that integrated with other theories have been applied by some researchers, including the intuitionistic fuzzy sets [2]-[6], fuzzy multiset [7]-[9] and fuzzy soft sets[10]-[14]. Torra and Norakawa [15]-[16] introduced hesitant fuzzy sets (HFSs) in which the membership of an object to a concept is presented by a series of some different values between 0 to 1 . HFSs can mirror human's hesitancy further objectively than the other usual extensions of fuzzy sets. Since its introduction, researchers have used it to answer numerous decision-making issues [17]-[38]. Aside from that, some researchers integrated HFSs with some other extensions of fuzzy sets. Lv et al. [39] studied on hesitant fuzzy information measures and their clustering application. Xu \& Zhang [40] made an overview on the applications of the hesitant fuzzy sets in group decision-making.

Babitha and John [41] first studied the hesitant fuzzy soft sets (HFSSs) which are the hybrid structure between HFSs and fuzzy soft set. They proposed the basic operation of HFSSs such as union, intersection, complement and proved the De Morgan's law. Consequently, Wang et al. [42] proposed HFSSs and their operations such as "AND", "OR" complement, union and intersection and their basic law properties. Beg and Rashid [43] presented the idea of an HFSSs where adaptation to manage the conditions in which experts assess an alternative giving to finite criteria in all possible values. They also proposed the distance measure between any two elements of the HFSSs. Rezaei andRezaei [44] proposed distance and similarity measures for HFSSs by using well-known Hamming, Euclidean, and Minkowski distance measures while Li et al. [45] extended the concept of HFSS to generalized HFSSs.

Among the significant milestones in the development of hesitant fuzzy soft sets and their generalizations is the introduction of the fuzzy parameterized aspect. The fuzzy parameterized aspect was firstly established by Cagman et al. [46] who proposed the fuzzy parameterized fuzzy soft (FPFS) sets and their basic operations and followed by others.[47]-[54] .

Characteristics of this work are as follows:

1. We extend the definitions of HFSS [41], [42] to the fuzzy parameterized, allowing this theory to be enhanced by weighting each parameter, namely 
FPHFSSs. We investigate certain connections between two FFPHFLTSSs and certain plain, binary-based set operations for FPHFSSs. There is also a mention of the property of operator.

2. Some of the previous methods added the maximum value, minimum value or any value to the shorter one until both have the same HFE length. These methods remove and alter data knowledge from the original data structure. In order to fill this gap, the TOPSIS algorithm is presented based on the FPHFSS's arithmetical mean and geometry mean without adding an element to HFE. This approach is simple and easy to understand.

The presentation of this article is as follows. In section 2, we call some basic concepts of hesitant fuzzy sets, fuzzy soft sets and hesitant fuzzy soft sets. In section 3, we proposed the concept of fuzzy parameterized hesitant fuzzy soft set (FPHFSSs) which is the combination of hesitant fuzzy soft set and fuzzy parameterized in which we provide the degree of importance for each alternative. We also expressed the proposed concept's basic operations namely intersection, union, and complement and then study some of their properties. In section 4, we introduce the TOPSIS based score index of FPHFSSs. Then we give numerical example of the FPHFSSs in solving decision-making problem and make a comparison analysis with other existing methods. Finally, we give the conclusion of our study and recommendation for further research.

\section{Preliminaries}

In this section, we recall some basic notions and definitions of hesitant fuzzy set and fuzzy soft set that will be used in this paper.

Definition 1([15]). Let a set $X$ be fixed. Then a hesitant fuzzy set (HFS) $H$ on $X$ in terms of a function $h$ is that when applied to $X$ a subset of $[0,1]$ return. To be easily understood $\mathrm{Xu}$ and Xia expressed the hesitant fuzzy set by

$$
H(x)=\left\{\left(x, h_{A}(x)\right): x \in X\right\}
$$

where $h_{A}(x)$ is a set of some different values in $[0,1]$ is called hesitant fuzzy elements (HFEs), representing the possible membership degrees of the element $x \in X$ to $A$. For three HFEs $h, h_{1}$ and $h_{2}$, some operations can be described as follows:

a) Lower bound: $h^{-}(x)=\min h(x)$

b) Upper bound: $h^{+}(x)=\max h(x)$

c) Complement: $h^{c}(x)=\{1-\gamma \mid \gamma \in h(x)\}$;

d) Union:

$\left(h_{1} \cup h_{2}\right)(x)=\left\{\gamma \in h_{1}(x) \cup h_{2}(x) \mid \gamma \geq \max \left\{h_{1}^{-}(x), h_{2}^{-}(x)\right\}\right\}$

e) Intersection:

$\left(h_{1} \cap h_{2}\right)(x)=\left\{\gamma \in h_{1}(x) \cap h_{2}(x) \mid \gamma \leq \min \left\{h_{1}^{+}(x), h_{2}^{+}(x)\right\}\right\}$
$\mathrm{Xu}$ and $\mathrm{Xia}$ [55] gave other forms of union and intersection of HFEs as below:

a) Union:

$$
\left(h_{1} \cup h_{2}\right)(x)=\cup_{\gamma_{1} \in h_{1}, \gamma_{2} \in h_{2}} \mid \max \left\{\gamma_{1}, \gamma_{2}\right\}
$$

b) Intersection:

$$
\left(h_{1} \cap h_{2}\right)(x)=\bigcup_{\gamma_{1} \in h_{1}, \gamma_{2} \in h_{2}} \mid \min \left\{\gamma_{1}, \gamma_{2}\right\}
$$

$\mathrm{Xia}$ and $\mathrm{Xu}$ [56] defined the HFEs of $h, h_{1}$ and $h_{2}$ as follows:

$$
\begin{array}{ll}
\text { c) } & h^{\lambda}(x)=\bigcup_{\gamma \in h}\left\{\gamma^{\lambda}\right\} \\
\text { d) } & \lambda h(x)=\bigcup_{\gamma \in h}\left\{1-\left(1-\gamma^{\lambda}\right)\right\} \\
\text { e) } & \left(h_{1} \oplus h_{2}\right)(x)=\bigcup_{\gamma_{1} \in h_{1}, \gamma_{2} \in h_{2}}\left\{\gamma_{1}+\gamma_{2}-\gamma_{1} \gamma_{2}\right\} \\
\text { f) } & \left(h_{1} \otimes h_{2}\right)(x)=\bigcup_{\gamma_{1} \in h_{1}, \gamma_{2} \in h_{2}}\left\{\gamma_{1} \gamma_{2}\right\}
\end{array}
$$

Definition 2([57]). Let $h=\left\{h_{1}, h_{2}, \ldots, h_{n}\right\}$ be an HFE. The following functions can be considered as the score index for HFEs:

The arithmetic mean score index:

$$
S_{A M}(h)=\frac{1}{n} \sum_{1=i}^{n} h_{i}
$$

The geometric-mean score index:

$$
S_{G M}(h)=\left(\prod_{1=i}^{n} h_{i}\right)^{\frac{1}{n}}
$$

Definition 3 ([58]).Let $U$ be an initial universe set and $E$ be universe set of parameters. A pair $\langle F, A\rangle$ is called a fuzzy soft set over $U$ where $F: A \rightarrow \tilde{P}(U)$ is a mapping from $A$ into $\tilde{P}(U)$. Here $\tilde{P}(U)$ denotes the power set of all fuzzy soft set on $U$ and $A \subseteq E$.

Definition 4 ([41]).Let $U$ be an initial universe set and $E$ be universe set of parameters. A pair $(F, E)$ is called a hesitant fuzzy soft set (HFSS) over U, if and only if $F: E \rightarrow H(U)$ defined as

$$
F(x)=\left\{x, \varpi_{K}(x): x \in E\right\}
$$

Where $H(U)$ is the set of all hesitant fuzzy subset of $U$ over the set $E, \varpi_{K}(x)$ is the hesitant degree of membership of $x$ over the parameter $x \in E$.

\section{Fuzzy Parameterized Hesitant Fuzzy Soft Set}

In this section, we shall define fuzzy parameterized hesitant fuzzy soft set and their operations with examples.

Definition 5. Let $U$ be an initial universe, $E$ the set of all parameters and $K$ a fuzzy set over $E$ with membership function $\mu_{K}: E \rightarrow[0,1]$ and let $\varpi_{K}$ be a hesitant fuzzy 
set over $U$ for all $x \in E$. Then a fuzzy parameterized hesitant fuzzy soft sets (FPHFSSs) over $U$ is a set defined by function $\zeta_{K}$ representing a mapping

$$
\zeta_{K}=\left\{\left(\frac{x}{\mu_{K}(x)}, \varpi_{K}(x)\right): x \in E, \varpi_{K}(x) \in H(x), \mu_{K}(x) \in[0,1]\right\}
$$

It should be noted that the set of all FPHFSs over $U$ will be denoted by FPHFSS(U).

Example 1. Let $U=\left\{h_{1}, h_{2}, h_{3}, h_{4}\right\}$ be four houses, $E=\left\{x_{1}, x_{2}, x_{3}\right\}$ be a set of parameters $x_{1}$ for the price, $x_{2}$ for the location and $x_{3}$ for the size. Suppose $K=\left\{\frac{x_{1}}{0.4}, \frac{x_{2}}{0.8}, \frac{x_{3}}{0.2}\right\}$ and $\varpi\left(x_{i}\right)$ are defined such that $\varpi_{K}(x)=\varnothing$ if $\mu_{K}(x)=0$. Suppose,

$$
\varpi_{K}\left(x_{1}\right)=\left\{\frac{h_{1}}{\{0,2,0.3\}}, \frac{h_{2}}{\{0.5,0.6\}}, \frac{h_{3}}{\{0.3\}}, \frac{h_{4}}{\{0.3,0.5\}}\right\}
$$

$\varpi_{K}\left(x_{2}\right)=\left\{\frac{h_{1}}{\{0.4,0.6,0.7\}}, \frac{h_{2}}{\{0.5,0.7,0.8\}}, \frac{h_{3}}{\{0.6,0.8\}}, \frac{h_{4}}{\{0.7,0.9\}}\right\}$

$$
\varpi_{K}\left(x_{3}\right)=\left\{\frac{h_{1}}{\{0,2,0.4\}}, \frac{h_{2}}{\{0.6,0.7\}}, \frac{h_{3}}{\{0.8,0.9\}}, \frac{h_{4}}{\{0.3,0.5\}}\right\}
$$

Then the FPHFSS set is given by

$$
\begin{aligned}
\zeta_{K}= & \left\{\left(\frac{x_{1}}{0.4},\left\{\frac{h_{1}}{\{0,2,0.3\}}, \frac{h_{2}}{\{0.5,0.6\}}, \frac{h_{3}}{\{0.3\}}, \frac{h_{4}}{\{0.3,0.5\}}\right\}\right),\right. \\
& \left(\frac{x_{2}}{0.8},\left\{\frac{h_{1}}{\{0.4,0.6,0.7\}}, \frac{h_{2}}{\{0.5,0.7,0.8\}}, \frac{h_{3}}{\{0.7,0.8\}}, \frac{h_{4}}{\{0.7,0.9\}}\right\}\right), \\
& \left.\left(\frac{x_{3}}{0.2},\left\{\frac{h_{1}}{\{0.2,0.4\}}, \frac{h_{2}}{\{0.6,0.7\}}, \frac{h_{3}}{\{0.8,0.9\}}, \frac{h_{4}}{\{0.3,0.5\}}\right\}\right)\right\}
\end{aligned}
$$

Definition 6. Two FPHFSSs $\zeta_{K}$ and $\zeta_{L}$ are said to be equal if $\zeta_{K}$ is a subset of $\zeta_{L}$ and $\zeta_{L}$ is a subset of $\zeta_{K}$. In other words $\zeta_{K}=\zeta_{L}$.

Definition 7. Two FPHFSSs are said to be equal, and we write $\zeta_{K}=\zeta_{L}$ if $\zeta_{K}$ is an FPHFSS-subset of $\zeta_{L}$ and $\zeta_{L}$ is an FPHSS subset of $\zeta_{K}$. In other words, $\zeta_{K}=\zeta_{L}$ if the following conditions are satisfied:

$$
\begin{array}{ll}
\text { 1. } & \mu_{K}(x)=\mu_{L}(x), \forall x \in E ; \\
\text { 2. } & \varpi_{K}(x)=\varpi_{L}(x), \forall x \in E .
\end{array}
$$

Definition 8. Let $\zeta_{K}$ be an FPHFSS. If $\zeta_{k}(x)=\phi$ then $\zeta_{K}$ is called an empty FPHFSSs denoted by $\zeta_{\phi}$ for all $x \in K$.

Definition 9. Let $\zeta_{K}$ be an FPHFSS. If $\zeta_{K}(x)=\breve{1}$. Then $\zeta_{K}$ is called a full FPHFSS set denoted by $\zeta_{E}$ for all $x \in K$.

Proposition 1. Let $\zeta_{K}, \zeta_{L}$ and $\zeta_{M}$ be any three of FPHFSSs. Then the following results hold:

1. $\zeta_{K} \subseteq \zeta_{E}$,

$$
\begin{array}{ll}
\text { 2. } & \zeta_{\phi} \subseteq \zeta_{K}, \\
\text { 3. } & \zeta_{K} \subseteq \zeta_{K}, \\
\text { 4. } & \zeta_{K} \subseteq \zeta_{L} \text { and } \zeta_{L} \subseteq \zeta_{M} \text { then } \zeta_{K} \subseteq \zeta_{M}, \\
\text { 5. } & \zeta_{K}=\zeta_{L} \text { and } \zeta_{L}=\zeta_{M} \text { then } \zeta_{M}=\zeta_{K}, \\
\text { 6. } & \zeta_{K} \subseteq \zeta_{L} \text { and } \zeta_{L} \subseteq \zeta_{K} \text { then } \zeta_{K}=\zeta_{L},
\end{array}
$$

Proof: The proof is straightforward.

Definition 10. Let $\zeta_{K}$ be an FPHSS. Then the complement of $\zeta_{K}=\left\{\left(\frac{x}{\mu_{K}(x)}, \varpi_{K}(x)\right)\right\}$ is denoted by $\zeta_{K}{ }^{c}=\left\{\left(\frac{x}{\mu_{K}(x)}, \varpi_{K}(x)\right)\right\}^{c}$ defined by $\mu_{K^{c}}(x)=1-\mu_{K}(x)$ and $\left.\varpi_{K^{\check{c}}}(x)\right)=1-\varpi_{K}(x)$ where $\mathrm{c}$ is a fuzzy complement and $\breve{c}$ is a hesitant fuzzy soft complement.

Example 2. Consider example 1. By using Definition 1, we have

$$
\begin{aligned}
\zeta_{K}{ }^{c}= & \left\{\left(\frac{x_{1}}{0.6}\left\{\frac{h_{1}}{\{0.8,0.7\}}, \frac{h_{2}}{\{0.5,0.4\}}, \frac{h_{3}}{\{0.7\}}, \frac{h_{4}}{\{0.7,0.5\}}\right\}\right),\right. \\
& \left(\frac{x_{2}}{0.2},\left\{\frac{h_{1}}{\{0.6,0.4,0.3\}}, \frac{h_{2}}{\{0.5,0.3,0.2\}}, \frac{h_{3}}{\{0.4,0.2\}}, \frac{h_{4}}{\{0.3,0.1\}}\right\}\right), \\
& \left.\left(\frac{x_{3}}{0.8},\left\{\frac{h_{1}}{\{0.8,0.6\}}, \frac{h_{2}}{\{0.4,0.3\}}, \frac{h_{3}}{\{0.2,0.1\}}, \frac{h_{4}}{\{0.7,0.5\}}\right\}\right)\right\}
\end{aligned}
$$

Proposition 2 Let $\zeta_{K}$ be an FPHSS. Then the following results hold:

$$
\begin{aligned}
& \text { 1. }\left(\zeta_{K}{ }^{c}\right)^{c}=\zeta_{K} \\
& \text { 2. } \quad \zeta_{\phi}{ }^{c}=\zeta_{E}
\end{aligned}
$$

Proof: The proof is straightforward.

Definition 11. Let $\zeta_{K}=\left\{\frac{x}{\mu_{K}(x)}, \varpi_{K}(x)\right\} \quad$ and $\zeta_{L}=\left\{\frac{x}{\mu_{L}(x)}, \varpi_{L}(x)\right\}$ be two FPHFSSs. The union of $\zeta_{K}$ and $\zeta_{L}$ denoted by $\zeta_{K} \cup \zeta_{L}$ is defined by

$$
\mu_{K \cup L}(x)=\max \left(\mu_{K}(x), \mu_{L}(x)\right)
$$

and

$$
\varpi_{K \breve{L}}(x)=\max \left(\varpi_{K}(x), \varpi_{L}(x)\right)
$$

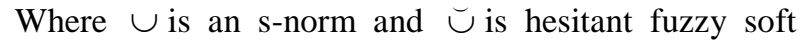
union based on Definition 1 in $\mathrm{f}$ ).

Example 3. Consider $\zeta_{K}$ as in Example 1 and let $\zeta_{L}$ be another FPHFSS defined as follows: 


$$
\begin{aligned}
\zeta_{L}= & \left\{\left(\frac{x_{1}}{0.3},\left\{\frac{h_{1}}{\{0.3,0.4,0.5\}}, \frac{h_{2}}{\{0.4,0.5,0.6\}}, \frac{h_{3}}{\{0.1,0.2,0.3\}}, \frac{h_{4}}{\{0.5\}}\right\}\right),\right. \\
& \left(\frac{x_{2}}{0.6},\left\{\frac{h_{1}}{\{0.2,0.4\}}, \frac{h_{2}}{\{0.3,0.4,0.5\}}, \frac{h_{3}}{\{0.7\}}, \frac{h_{4}}{\{0.5,0.7\}}\right\}\right), \\
& \left.\left(\frac{x_{3}}{0.4},\left\{\frac{h_{1}}{\{0.2,0.4,0.5\}}, \frac{h_{2}}{\{0.3\}}, \frac{h_{3}}{\{0.4,0.6\}}, \frac{h_{4}}{\{0.6,0.7,0.8\}}\right\}\right)\right\}
\end{aligned}
$$

The intersection of $\zeta_{K}$ and $\zeta_{L}$ denoted by $\zeta_{K} \cup \zeta_{L}$ following Definition 11 is given as

$$
\begin{aligned}
& \zeta_{K \cup L}(x)=\left\{\left(\frac{x_{1}}{0.4},\left\{\frac{h_{1}}{\{0.3,0.4,0.5\}}, \frac{h_{2}}{\{0.5,0.6\}}, \frac{h_{3}}{\{0.3\}}, \frac{h_{4}}{\{0.5\}}\right\}\right),\right. \\
& \left(\frac{x_{2}}{0.8},\left\{\frac{h_{1}}{\{0.4,0.6,0.7\}}, \frac{h_{2}}{\{0.5,0.7,0.8\}}, \frac{h_{3}}{\{0.7,0.8\}}, \frac{h_{4}}{\{0.7,0.9\}}\right\}\right), \\
& \left.\left(\frac{x_{3}}{0.4},\left\{\frac{h_{1}}{\{0.2,0.4,0.5\}}, \frac{h_{2}}{\{0.6,0.7\}}, \frac{h_{3}}{\{0.8,0.9\}}, \frac{h_{4}}{\{0.6,0.7,0.8\}}\right\}\right)\right\}
\end{aligned}
$$

Proposition 3. Let $\zeta_{K}$ and $\zeta_{L}$ be any two FPHFSSs. Then the following results hold:
1. $\zeta_{K} \cup \zeta_{K}=\zeta_{K}$
2. $\zeta_{\phi} \cup \zeta_{K}=\zeta_{K}$
3. $\zeta_{K} \cup \zeta_{E}=\zeta_{E}$
4. $\zeta_{K} \cup \zeta_{L}=\zeta_{L} \cup \zeta_{K}$

Proof. The proof is straightforward.

Definition 12. Let $\zeta_{K}=\left\{\frac{x}{\mu_{K}(x)}, \varpi_{K}(x)\right\} \quad$ and $\zeta_{L}=\left\{\frac{x}{\mu_{L}(x)}, \varpi_{L}(x)\right\}$ be two FPHFSSs. The intersection of $\zeta_{K}$ and $\zeta_{L}$ which is denoted by $\zeta_{K} \cap \zeta_{L}$ is defined by

$$
\mu_{K \cap L}(x)=\min \left(\mu_{K}(x), \mu_{L}(x)\right)
$$

and

$$
\varpi_{K \check{ } \check{L}}(x)=\min \left(\varpi_{K}(x), \varpi_{L}(x)\right)
$$

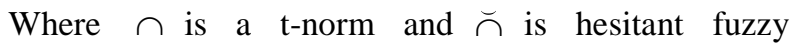
intersection based on Definition 1 in $\mathrm{g}$ ).

Example 4. Let $\zeta_{K}$ as in Example 1 and $\zeta_{L}$ as in Example 3 be two FPHFSSs. The intersection of $\zeta_{K}$ and $\zeta_{L}$ which is denoted by $\zeta_{K} \cap \zeta_{L}$ following Definition 12 is given as

$$
\begin{aligned}
& \zeta_{K \cap L}=\left\{\left(\frac{x_{1}}{0.3},\left\{\frac{h_{1}}{\{0.2,0.3\}}, \frac{h_{2}}{\{0.4,0.5,0.6\}}, \frac{h_{3}}{\{0.1,0.2,0.3\}}, \frac{h_{4}}{\{0.3,0.5\}}\right\}\right),\right. \\
& \left(\frac{x_{2}}{0.6},\left\{\frac{h_{1}}{\{0.2,0.4\}}, \frac{h_{2}}{\{0.3,0.4,0.5\}}, \frac{h_{3}}{\{0.7\}}, \frac{h_{4}}{\{0.5,0.7\}}\right\}\right), \\
& \left.\left(\frac{x_{3}}{0.2},\left\{\frac{h_{1}}{\{0.2,0.4\}}, \frac{h_{2}}{\{0.3\}}, \frac{h_{3}}{\{0.4,0.6\}}, \frac{h_{4}}{\{0.3,0.5\}}\right\}\right)\right\}
\end{aligned}
$$

Proposition 4. Let $\zeta_{K}, \zeta_{L}$ and $\zeta_{M}$ be any three of FPHFSSs. Then the following results hold:
1. $\zeta_{K} \cap \zeta_{K}=\zeta_{K}$
2. $\zeta_{\phi} \cap \zeta_{K}=\zeta_{\phi}$
3. $\zeta_{K} \cap \zeta_{E}=\zeta_{K}$
4. $\zeta_{K} \cap \zeta_{L}=\zeta_{L} \cap \zeta_{K}$

Proof: The proof is straightforward.

Proposition 5. Let $\zeta_{K}, \zeta_{L}$ and $\zeta_{M}$ be any three of FPHFSSs. Then the following results hold:

$$
\begin{aligned}
& \text { 1. } \quad\left(\zeta_{K} \cup \zeta_{K}\right)^{c}=\zeta_{K}{ }^{c} \\
& \text { 2. } \quad\left(\zeta_{K} \cap \zeta_{L}\right)^{c}=\zeta_{K}{ }^{c} \cup \zeta_{L}{ }^{c}
\end{aligned}
$$

Proof: The proof is straightforward.

Definition 13. The operationAND for two FPHFSSs $\zeta_{K}$ and $\zeta_{L}$ which is denoted by $\zeta_{K} \wedge \zeta_{L}$ is defined by $\zeta_{K} \wedge \zeta_{L}=\zeta_{K} \cap \zeta_{L}$.

Definition 14. The operationOR for two FPHFSSs $\zeta_{K}$ and $\zeta_{L}$ which is denoted by $\zeta_{K} \vee \zeta_{L}$ is defined by $\zeta_{K} \vee \zeta_{L}=\zeta_{K} \cup \zeta_{L}$.

Theorem 4. (De Morgan Law of FPHFSSs).

Let $\zeta_{K}$ and $\zeta_{L}$ be two FPHFSSs over $U$; we have

$$
\begin{aligned}
& \text { 1. } \quad\left(\zeta_{K} \vee \zeta_{L}\right)^{c}=\zeta_{K}{ }^{c} \wedge \zeta_{L}{ }^{c}, \\
& \text { 2. } \quad\left(\zeta_{K} \wedge \zeta_{L}\right)^{c}=\zeta_{K}{ }^{c} \vee \zeta_{K}{ }^{c},
\end{aligned}
$$

Proof:

Note that,

$$
\begin{aligned}
\mu_{(K \cup L)^{c}}(x) & =\left(\mu_{(K \cup L)}(x)\right)^{c} \\
& =1-\max \left(\mu_{K}(x), \mu_{L}(x)\right) \\
& =\min \left(\left(1-\mu_{K}(x),\left(1-\mu_{L}(x)\right)\right.\right. \\
& =\min \left(\mu_{K^{c}}(x), \mu_{L^{c}}(x)\right) \\
& =\mu_{K^{c} \cap L^{c}}(x)
\end{aligned}
$$

and

$$
\begin{aligned}
\varpi_{(K \cup L)^{c}}(x) & =\left(\varpi_{(K \cup L)}(x)\right)^{c} \\
& =1-\max \left(\varpi_{K}(x), \varpi_{L}(x)\right) \\
& =\min \left(\left(1-\varpi_{K}(x),\left(1-\varpi_{L}(x)\right)\right.\right. \\
& =\min \left(\varpi_{K^{\check{c}}}(x), \varpi_{L^{\check{c}}}(x)\right) \\
& =\varpi_{K^{\bar{c}} \cap L^{\check{c}}}(x)
\end{aligned}
$$

Theorem 5. (Associative Law of FPHFSSs)

Let $\zeta_{K}, \zeta_{L}$ and $\zeta_{M}$ be any three FPHFSSs over $U$. Then the following results hold:

1. $\zeta_{K} \wedge\left(\zeta_{L} \wedge \zeta_{M}\right)=\left(\zeta_{K} \wedge \zeta_{L}\right) \wedge \zeta_{M}$

2. $\zeta_{K} \vee\left(\zeta_{L} \vee \zeta_{M}\right)=\left(\zeta_{K} \vee \zeta_{L}\right) \vee \zeta_{M}$

Proof:

Note that,

$$
\mu_{K} \wedge\left(\mu_{L} \wedge \mu_{M}\right)=\left(\mu_{K} \wedge \mu_{L}\right) \wedge \mu_{M}
$$

and 


$$
\varpi_{K} \wedge\left(\varpi_{L} \wedge \varpi_{M}\right)=\left(\varpi_{K} \wedge \varpi_{L}\right) \wedge \varpi_{M}
$$

so that

$$
\zeta_{K} \wedge\left(\zeta_{L} \wedge \zeta_{M}\right)=\left(\zeta_{K} \wedge \zeta_{L}\right) \wedge \zeta_{M}
$$

Similarly

$$
\mu_{K} \vee\left(\mu_{L} \vee \mu_{M}\right)=\left(\mu_{K} \vee \mu_{L}\right) \vee \mu_{M}
$$

and

$$
\varpi_{K} \vee\left(\varpi_{L} \vee \varpi_{M}\right)=\left(\varpi_{K} \vee \varpi_{L}\right) \vee \varpi_{M}
$$

so that

$$
\zeta_{K} \vee\left(\zeta_{L} \vee \zeta_{M}\right)=\left(\zeta_{K} \vee \zeta_{L}\right) \vee \zeta_{M}
$$

Theorem 6. (Distributive Law of FPHFSSs).

Let $\zeta_{K}, \zeta_{L}$ and $\zeta_{M}$ be any three FPHFSSs over $U$. Then the following results hold:

$$
\begin{array}{ll}
\text { 1. } & \zeta_{K} \wedge\left(\zeta_{L} \vee \zeta_{M}\right)=\left(\zeta_{K} \wedge \zeta_{L}\right) \vee\left(\zeta_{K} \wedge \zeta_{M}\right) \\
\text { 2. } & \zeta_{K} \vee\left(\zeta_{L} \wedge \zeta_{M}\right)=\left(\zeta_{K} \vee \zeta_{L}\right) \wedge\left(\zeta_{K} \vee \zeta_{M}\right)
\end{array}
$$

Proof:

Note that,

$$
\mu_{K} \wedge\left(\mu_{L} \vee \mu_{M}\right)=\left(\mu_{K} \wedge \mu_{L}\right) \vee\left(\mu_{K} \wedge \mu_{M}\right)
$$

and

$$
\varpi_{K} \wedge\left(\varpi_{L} \vee \varpi_{M}\right)=\left(\varpi_{K} \wedge \varpi_{L}\right) \vee\left(\varpi_{K} \wedge \varpi_{M}\right)
$$

so that

$$
\zeta_{K} \wedge\left(\zeta_{L} \vee \zeta_{M}\right)=\left(\zeta_{K} \wedge \zeta_{L}\right) \vee\left(\zeta_{K} \wedge \zeta_{M}\right)
$$

Similarly

$$
\mu_{K} \vee\left(\mu_{L} \wedge \mu_{M}\right)=\left(\mu_{K} \vee \mu_{L}\right) \wedge\left(\mu_{K} \vee \mu_{M}\right)
$$

and

$$
\varpi_{K} \vee\left(\varpi_{L} \wedge \varpi_{M}\right)=\left(\varpi_{K} \vee \varpi_{L}\right) \wedge\left(\varpi_{K} \vee \varpi_{M}\right)
$$

so that

$$
\zeta_{K} \vee\left(\zeta_{L} \wedge \zeta_{M}\right)=\left(\zeta_{K} \vee \zeta_{L}\right) \wedge\left(\zeta_{K} \vee \zeta_{M}\right)
$$

\section{Applications}

In this part, we will explain how to utilize this algorithm in solving MCDM. We extend the theory of HFSSs by giving importance weight for each parameter in decision making process. We utilize the TOPSIS algorithm with arithmetic mean and geometric mean score to solve problem in FPHFSSs environment. It should be noted that some of the existing operations, arrangement and various measures of hesitant fuzzy sets need the hesitant fuzzy elements to have exactly the same length. In practice, however, the length of the hesitant fuzzy element may vary. The approach proposed in [56] applied those elements to the shorter hesitant fuzzy element, rendering its equivalent to another hesitant fuzzy element, or repeating its elements to obtain two sequence of the same length. These approaches would break the original data structure and alter the data details [39]. In this paper, we presented an algorithm based on the two score functions of HFE by Farhadinia [57]. Based on this argument, we materialize with our algorithm for FPHFSS given as below.

Algorithm: The TOPSIS based score index of FPHFSSs.

Step 1. Transform the FPHFSSs into fuzzy decision matrix. The FPHFSSs matrix of decision $K$ can be represented as

$$
K=\left[\begin{array}{cccc}
\mu_{1} h_{11} & \mu_{2} h_{12} & \cdots & \mu_{n} h_{1 n} \\
\mu_{1} h_{21} & \mu_{1} h_{22} & \cdots & \mu_{n} h_{2 n} \\
\vdots & \vdots & \ddots & \vdots \\
\mu_{1} h_{m 1} & \mu_{1} h_{m 2} & \cdots & \mu_{n} h_{m n}
\end{array}\right]
$$

Step 2. Calculate the score index matrix of the hesitant fuzzy element of FPHFSSs.

the arithmetic mean score

$$
\widehat{S}_{A M}=\left(\frac{1}{n} \sum_{i}^{i=l} \mu h_{i}\right)^{\psi\left(h_{i}\right)}
$$

the geometry mean score

$$
\hat{S}_{G M}=\left(\left(\prod_{i=1}^{l} \mu h_{i}\right)^{\frac{1}{l}}\right)^{\psi}
$$

where $l$ is the number of elements in HFE and $\psi\left(h_{i}\right)=1-\frac{1}{l\left(h_{i}\right)}$ is a value of hesitant degree.

Step 3. Determine the positive ideal solution (PIS) and negative ideal solution (NIS)

$$
A^{+}=\left\{x_{j,} \max \left\langle\widehat{S}_{A M_{i j}}\right\rangle \mid j=1,2, \ldots, n\right\}(5)
$$

and

$$
A^{-}=\left\{x_{j}, \min \left\langle\widehat{S}_{A M_{i j}}\right\rangle \mid j=1,2, \ldots, n\right\}(6)
$$

Step 4. Calculate distance from the PIS and NIS to score values.

$$
d_{i}^{+}=\sqrt{\sum_{j=1}^{n}\left|A^{+}{ }_{j}-\widehat{S}_{A M_{i j}}\right|^{2}} \quad, \mid i=1,2, \ldots, n(7)
$$

and

$$
d_{i}^{-}=\sqrt{\sum_{j=1}^{n} \mid \hat{S}_{A M_{i j}}-A^{-}{ }_{j}^{2}}, \mid i=1,2, \ldots, n(8)
$$

Step 5. Calculate the CI of every alternative

$$
C I_{i}=\frac{d_{i}^{-}}{d_{i}^{-}+d_{i}^{+}}
$$

Step 6. Rank the alternatives. 
Table 1. Fuzzy decision matrix of FPHFSSs

\begin{tabular}{|l|l|l|l|l|}
\hline & $C_{1}$ & $C_{2}$ & $C_{3}$ & $C_{4}$ \\
\hline$P_{1}$ & $0.15\{0.3,0.4,0.5\}$ & $0.3\{0.1,0.7,0.8,0.9\}$ & $0.2\{0.2,0.4,0.5\}$ & $0.35\{0.3,0.5,0.6,0.9\}$ \\
\hline$P_{2}$ & $0.15\{0.3,0.5\}$ & $0.3\{0.2,0.5,0.6,0.7,0.9\}$ & $0.2\{0.1,0.5,0.5,0.8\}$ & $0.35\{0.3,0.4,0.7\}$ \\
\hline$P_{3}$ & $0.15\{0.6,0.7\}$ & $0.3\{0.6,0.9\}$ & $0.2\{0.3,0.5,0.7\}$ & $0.35\{0.4,0.6\}$ \\
\hline$P_{4}$ & $0.15\{0.3,0.4,0.7,0.8\}$ & $0.3\{0.2,0.4,0.7\}$ & $0.2\{0.1,0.8\}$ & $0.35\{0.6,0.8,0.9\}$ \\
\hline$P_{5}$ & $0.15\{0.1,0.3,0.6,0.7,0.9\}$ & $0.3\{0.4,0.6,0.7,0.8\}$ & $0.2\{0.7,0.8,0.9\}$ & $0.35\{0.3,0.6,0.7,0.9\}$ \\
\hline
\end{tabular}

\subsection{Numerical Examples}

This section provides a numerical illustration of the viability of the proposed score index-TOPSIS approach (modified from Xu \& Xia, 2011a) in the FPHFSSs decision-making problems.A comparative analysis is provided to validate its reasonableness and usefulness with other existing methods.

Energy is an important element for community social and economic growth. Considering five energy projects to be invested $P_{i}(i=1,2,3,4,5)$ the decision-makers will assess five potential projects anonymously from the four following criteria, technological $\left(C_{l}\right)$, environmental $\left(C_{2}\right)$, socio-political $\left(C_{3}\right)$, and economic $\left(C_{4}\right)$. The modified part is where the degree of importance for each criteria is directly decided by the decision makers.

We present our methods to solve the MCDM problem above using the steps presented before

Step 1. Construct the FPHFSSs into fuzzy decision matrix as shown in Table 1.

Step 2. Find the score index of the hesitant fuzzy element of FPHFSSs according to eq.(3). The arithmetic mean score of FPHFSSs are shown in Table 2.

Table 2. Arithmetic mean score matrix of FPHFSSs

\begin{tabular}{|l|c|c|c|c|}
\hline & $C_{1}$ & $C_{2}$ & $C_{3}$ & $C_{4}$ \\
\hline $\boldsymbol{P}_{1}$ & 0.0814 & 0.2109 & 0.1025 & 0.2311 \\
\hline $\boldsymbol{P}_{2}$ & 0.0949 & 0.1940 & 0.1189 & 0.2106 \\
\hline $\boldsymbol{P}_{3}$ & 0.1209 & 0.2598 & 0.1260 & 0.2475 \\
\hline $\boldsymbol{P}_{4}$ & 0.0958 & 0.1718 & 0.1342 & 0.2932 \\
\hline $\boldsymbol{P}_{5}$ & 0.0889 & 0.2109 & 0.1724 & 0.2460 \\
\hline
\end{tabular}

Step 3. Define the PIS and NIS based on arithmetic mean score as shown in Table 3 using equation (5) and (6).

Table 3. PIS and Nis arithmetic mean

\begin{tabular}{|c|c|c|c|c|}
\hline & $\boldsymbol{C}_{\boldsymbol{1}}$ & $\boldsymbol{C}_{\boldsymbol{2}}$ & $\boldsymbol{C}_{\boldsymbol{3}}$ & $\boldsymbol{C}_{\boldsymbol{4}}$ \\
\hline $\boldsymbol{A}^{+}$ & 0.1209 & 0.2598 & 0.1724 & 0.2932 \\
\hline $\boldsymbol{A}^{-}$ & 0.0814 & 0.1718 & 0.1025 & 0.2106 \\
\hline
\end{tabular}

Step 4 and step 5. To determine the separation $d^{+}$and $d$ of each alternative $P_{i}$ from the PIS and the NIS, the eq.(7) and eq.(8) shall be used. Then, calculate the relative closeness CI of each alternative using Equation (9).

Table 4.Distance from PIS and NIS CI

\begin{tabular}{|c|c|c|c|}
\hline & $\mathbf{d}^{+}$ & $\mathbf{d}^{-}$ & CI \\
\hline $\boldsymbol{P}_{\boldsymbol{1}}$ & 0.1127 & 0.0442 & 0.4360 \\
\hline $\boldsymbol{P}_{\boldsymbol{2}}$ & 0.1212 & 0.0308 & 0.2846 \\
\hline $\boldsymbol{P}_{3}$ & 0.0651 & 0.1059 & 1.7334 \\
\hline $\boldsymbol{P}_{\boldsymbol{4}}$ & 0.0992 & 0.0896 & 0.9935 \\
\hline $\boldsymbol{P}_{5}$ & 0.0751 & 0.0879 & 1.2578 \\
\hline
\end{tabular}

Step 6. Based on the value of $\mathrm{CI}$ the ranking is $P_{3} \succ P_{5} \succ P_{4} \succ P_{1} \succ P_{2}$.

\subsection{Comparison Analysis}

In this section, we will compare the ranking results with other existing methods. Table 5 compares the ranking of alternatives given by our proposed method and other existing methods. 
Table 5. The comparison ranking of alternatives

\begin{tabular}{|c|c|}
\hline Methods & Ranking \\
\hline Proposed methods & $\begin{array}{l}P_{3} \succ P_{5} \succ P_{4} \succ P_{1} \succ P_{2} \\
\text { TOPSIS - arithmetic mean. } \\
P_{3} \succ P_{5} \succ P_{4} \succ P_{1} \succ P_{2} \\
\text { TOPSIS - geometry mean. }\end{array}$ \\
\hline Liu and Wang [59] & $\begin{array}{l}P_{3} \succ P_{2} \succ P_{4} \succ P_{1} \succ P_{5} \\
\text { The preference weighted generalize distance with distance parameter }=1 \text { and preference parameter }=0.1 \\
P_{3} \succ P_{2} \succ P_{1} \succ P_{4} \succ P_{5} \\
\text { The preference weighted generalize distance with distance parameter }=2 \text { and preference parameter }=0.1 \\
P_{3} \succ P_{5} \succ P_{2} \succ P_{4} \succ P_{1} \\
\text { The preference weighted generalize distance with distance parameter }=6 \text { and preference parameter }=0.1\end{array}$ \\
\hline Sun et al. [22] & $\begin{array}{l}\text { Similarity like positive correlation decision making factor }=0.7 \text {. Weight of the distance like positive correlation } \\
\text { decision making factor }=0.6 \text {. } \\
P_{5} \succ P_{1} \succ P_{2} \succ P_{4} \succ P_{3}, \operatorname{Rc}(\eta) \\
P_{5} \succ P_{1} \succ P_{4} \succ P_{3} \succ P_{2}, \operatorname{Rc}(\delta) \\
P_{5} \succ P_{1} \succ P_{2} \succ P_{4} \succ P_{3} \operatorname{Rc}(\xi) \\
P_{5} \succ P_{1} \succ P_{2} \succ P_{4} \succ P_{3}, \operatorname{Rc}(\rho)\end{array}$ \\
\hline Li et al. [60] & $\begin{array}{l}\text { Weighted distance measure with preference. } \\
P_{5} \succ P_{3} \succ P_{4} \succ P_{1} \succ P_{2} \\
\text { for } \lambda=1, a=0.1, \beta=0.9 \\
P_{3} \succ P_{5} \succ P_{4} \succ P_{1} \succ P_{2} \\
\text { for } \lambda=1, a=0.5, \beta=0.5\end{array}$ \\
\hline $\mathrm{Xu}$ and $\mathrm{Xia}[55]$ & $\begin{array}{l}P_{3} \succ P_{5} \succ P_{4} \succ P_{1} \succ P_{2} \text { generalized hesitant weighted distance with } \lambda=1 \\
P_{3} \succ P_{5} \succ P_{4} \succ P_{1} \succ P_{2} \text { generalized hesitant weighted Hausdorff distance with } \lambda=1 \\
P_{3} \succ P_{5} \succ P_{4} \succ P_{1} \succ P_{2} \text { generalized hybrid hesitant weighted distance with } \lambda=1 .\end{array}$ \\
\hline
\end{tabular}

This table shows some of the rankings provided by our proposed methods and another established method of selecting the energy site. The distance between the hesitant evaluation values and the ideal reference intervals is uncertain on the basis of Liu and Wang [46]. For example, the hesitant evaluations for $\{0.7,0.6\}$ and $\{0.7,0.5\}$ with the ideal reference [ $0.7,0.5$ ] are the same even if the hesitant values are different. While the ranking given by Sun et al. [22] contradicts with the ranking from other methods, in which the $P_{3}$ is ranked in the final position although mostly $P_{3}$ is placed first by other methods. The ranking given by $\mathrm{Li}$ et al [60] is quite compatible with some other algorithms, even so their algorithms are complicated for decision-making because it is difficult to determine the value of $\lambda, a$ and $\beta$. Xu and $\mathrm{Xia}$ [55] presented the following rules for proper operation: the shorter one is expanded by inserting a minimum value, maximum value, or any value thereof until it has the same length as the longer one. The choice of this value depends mainly on the risk preferences of decision-makers. Optimists are expected to achieve desirable results and may bring maximum value and pessimists predict to contribute minimum value. Obviously, the original data structure will be broken and the data details modified [30].

\section{Conclusions}

In this paper, we consider the parameterized hesitant fuzzy soft set which includes the combination of the hesitant fuzzy set and fuzzy soft sets where an important degree is given for each element in the set of parameters. The introduction of elements of fuzzy parameters are used to avoid the degree of importance to which criteria are created from the rating of each alternative. Decision makers should decide which criteria are more important than others. Next, we study some of the algorithm's properties. The complement, union and intersection, AND and $O R$ operation have been defined on the FPHFSSs. In addition, the algorithm given can cater for certain methods which added or repeated the hesitant fuzzy elements to the same length as this technique could destroy the original information. Finally, we provided an example which demonstrates that this theory can be used to solve MCDM problems. Comparisons are made to show feasibility and viability of our proposed method. We hope that our work could enhance the study on hesitant fuzzy soft sets which could further be applied in many other areas such as data analysis and forecasting. At the same time, it is anticipated that the fuzzy parameterized concept can extend to other generalization of fuzzy sets such as interval fuzzy set, 
intuinistic fuzzy set, hesitant fuzzy set and others.

\section{REFERENCES}

[1] L. Zadeh, "Fuzzy Sets," Inf. Control, vol. 8, pp. 338-353, 1965, https://doi.org/10.1016/S0019-9958(65)90241-X.

[2] M. Kumar, "Evaluation of the intuitionistic fuzzy importance of attributes based on the correlation coefficient under weakest triangular norm and application to the hotel services," J. Intell. Fuzzy Syst., vol. 36, no. 4, pp. 3211-3223, 2019, doi: 10.3233/JIFS-18485.

[3] M. Kumar, "Intuitionistic fuzzy measures of correlation coefficient of intuitionistic fuzzy numbers under weakest triangular norm," Int. J. Fuzzy Syst. Appl., vol. 8, no. 1, pp. 48-64, 2019, doi: 10.4018/IJFSA.2019010103.

[4] R. Biswas, "Intuitionistic fuzzy theory for soft-computing: more appropriate tool than fuzzy theory," Int. J. Comput. Optim., vol. 6, no. 1, pp. 13-56, 2019, doi: 10.12988/ijco.2019.955.

[5] F. Feng, M. Liang, H. Fujita, R. R. Yager, and X. Liu, "Lexicographic orders of intuitionistic fuzzy values and their relationships," Mathematics, vol. 7, no. 2, pp. 1-26, 2019, doi: 10.3390/math7020166.

[6] Z. Jia and Y. Zhang, "Interval-Valued Intuitionistic Fuzzy Multiple Attribute Group Decision Making with Uncertain Weights," Math. Probl. Eng., vol. 2019, no. 3, 2019, doi: 10.1155/2019/5092147.

[7] A. Kandil, S. A. El-Sheikh, M. Hosny, and M. Raafat, "Hesitant fuzzy soft multisets and their applications in decision-making problems," Soft Comput., vol. 24, no. 6, pp. 4223-4232, 2020, doi: 10.1007/s00500-019-04187-w.

[8] A. Dockhorn, T. Schwensfeier, and R. Kruse, "Fuzzy Multiset Clustering for Metagame Analysis," Proc. 2019 Conf. Int. Fuzzy Syst. Assoc. Eur. Soc. Fuzzy Log. Technol. (EUSFLAT 2019), no. May, 2019, doi: 10.2991/eusflat-19.2019.74.

[9] R. Muthuraj and S. Devi, "New similarity measure between intuitionistic fuzzy multisets based on tangent function and its application in medical diagnosis," Int. J. Recent Technol. Eng., vol. 8, no. 2 Special Issue 3, pp. 161-165, 2019, doi: 10.35940/ijrte.B1030.0782S319.

[10] A. Nazra, Syafruddin, R. Lestari, and G. C. Wicaksono, "Hesitant intuitionistic fuzzy soft sets," J. Phys. Conf. Ser., vol. 890, no. 1, pp. 0-6, 2017, doi: 10.1088/1742-6596/890/1/012118.

[11] S. Habib and M. Akram, Diagnostic methods and risk analysis based on fuzzy soft information, vol. 11, no. 8 . 2018.

[12] V. Lambodharan and N. Anitha, "Interval valued fuzzy soft sets and fuzzy connectives," Int. J. Recent Technol. Eng., vol. 8, no. 4, pp. 20-23, 2019, doi: 10.35940/ijrte.C4315.1 18419 .

[13] M. J. Khan, P. Kumam, S. Ashraf, and W. Kumam,
"Generalized picture fuzzy soft sets and their application in decision support systems," Symmetry (Basel)., vol. 11, no. 3, pp. 1-27, 2019, doi: 10.3390/sym11030415.

[14] Z. Liu, K. Qin, and Z. Pei, "A Method for Fuzzy Soft Sets in Decision-Making Based on an Ideal Solution," Symmetry (Basel)., vol. 9, pp. 1-22, 2017, doi: 10.3390/sym9100246.

[15] V. Torra and Y. Narukawa, "On hesitant fuzzy sets and decision,” IEEE Int. Conf. Fuzzy Syst., pp. 1378-1382, 2009, doi: 10.1109/FUZZY.2009.5276884.

[16] V. Torra, "Hesitant Fuzzy Sets," Int. J. Intell. Syst., vol. 25, pp. 529-539, 2010, doi: 10.1002/int.

[17] K. Bisht and S. Kumar, "Fuzzy time series forecasting method based on hesitant fuzzy sets," Expert Syst. Appl., vol. 64, pp. 557-568, 2016, doi: 10.1016/j.eswa.2016.07.044.

[18] N. Chen, Z. Xu, and M. Xia, "Correlation coefficients of hesitant fuzzy sets and their applications to clustering analysis," Appl. Math. Model., vol. 37, no. 4, pp. 2197-2211, 2013, doi: 10.1016/j.apm.2012.04.031.

[19] F. Zhang, J. Li, J. Chen, J. Sun, and A. Attey, "Hesitant distance set on hesitant fuzzy sets and its application in urban road traffic state identification," Eng. Appl. Artif. Intell., vol. 61, no. August 2016, pp. 57-64, 2017, doi: 10.1016/j.engappai.2017.02.004.

[20] S. Sardari, M. Eftekhari, and F. Afsari, "Hesitant fuzzy decision tree approach for highly imbalanced data classification," Appl. Soft Comput. J., vol. 61, pp. 727-741, 2017, doi: 10.1016/j.asoc.2017.08.052.

[21] S. K. De and S. Sankar, "Paci fi c Science Review A: Natural Science and Engineering Multi-criterion multi-attribute decision-making for an EOQ model in a hesitant fuzzy environment," Pacific Sci. Rev. A Nat. Sci. Eng., vol. 17, no. 2, pp. 61-68, 2016, doi: 10.1016/j.psra.2015.11.006.

[22] G. Sun, X. Guan, X. Yi, and Z. Zhou, "An innovative TOPSIS approach based on hesitant fuzzy correlation coefficient and its applications," Appl. Soft Comput. J., vol. 68, pp. 249-267, 2018, doi: 10.1016/j.asoc.2018.04.004.

[23] Q. Li, Y. Diao, Z. Gong, and A. Hu, "Grey language hesitant fuzzy group decision making method based on kernel and grey scale," Int. J. Environ. Res. Public Health, vol. 15, no. 3, 2018, doi: 10.3390/ijerph15030436.

[24] Y. Wang, R. Zhang, and L. Qian, "An Improved A* Algorithm Based on Hesitant Fuzzy Set Theory for Multi-Criteria Arctic Route Planning," Symmetry (Basel)., vol. 10, no. 12, p. 765, 2018, doi: 10.3390/sym10120765.

[25] M. Ranjbar, S. Effati, and A. V. Kamyad, "T-operators in hesitant fuzzy sets and their applications to fuzzy rule-based classifier," Appl. Soft Comput. J., vol. 62, pp. 423-440, 2018, doi: 10.1016/j.asoc.2017.10.016.

[26] Z. Wu and J. Xu, "A consensus model for large-scale group decision making with hesitant fuzzy information and changeable clusters," Inf. Fusion, vol. 41, pp. 217-231, 2018, doi: 10.1016/j.inffus.2017.09.011.

[27] J. Li, J. qiang Wang, and J. hua Hu, "Consensus building for hesitant fuzzy preference relations with multiplicative consistency," Comput. Ind. Eng., vol. 128, no. December 
2018, pp. 387-400, 2019, doi: 10.1016/j.cie.2018.12.051.

[28] M. Sarwar Sindhu, T. Rashid, and M. Khan, "Group decision making based on hesitant fuzzy ranking of hesitant fuzzy preference relations," J. Intell. Fuzzy Syst., vol. 37, no. 2, pp. 2563-2573, 2019, doi: 10.3233/JIFS-182780.

[29] H. Liao, Z. Xu, and X. J. Zeng, "Novel correlation coefficients between hesitant fuzzy sets and their application in decision making," Knowledge-Based Syst., vol. 82, pp. 115-127, 2015, doi: 10.1016/j.knosys.2015.02. 020 .

[30] J. C. R. Alcantud and A. Giarlotta, "Necessary and possible hesitant fuzzy sets: A novel model for group decision making," Inf. Fusion, vol. 46, pp. 63-76, 2019, doi: 10.1016/j.inffus.2018.05.005.

[31] M. Akram, A. Adeel, and J. C. R. Alcantud, "Hesitant fuzzy $\mathrm{N}$-soft sets: A new model with applications in decision-making," J. Intell. Fuzzy Syst., vol. 36, no. 6, pp. 6113-6127, 2019, doi: 10.3233/JIFS-181972.

[32] D. Li, W. Zeng, and Y. Zhao, "Note on distance measure of hesitant fuzzy sets," Inf. Sci. (Ny)., vol. 321, pp. 103-115, 2015, doi: 10.1016/j.ins.2015.03.076.

[33] Z. Mu, S. Zeng, and T. Baležentis, "A novel aggregation principle for hesitant fuzzy elements," Knowledge-Based Syst., vol. 84, pp. 134-143, 2015, doi: 10.1016/j.knosys.20 15.04.008.

[34] J. C. R. Alcantud, R. De Andrés Calle, and M. J. M. Torrecillas, "Hesitant Fuzzy Worth: An innovative ranking methodology for hesitant fuzzy subsets," Appl. Soft Comput. J., vol. 38, pp. 232-243, 2016, doi: 10.1016/j.asoc.2015.09 .035

[35] J. Qin, X. Liu, and W. Pedrycz, "Frank aggregation operators and their application to hesitant fuzzy multiple attribute decision making," Appl. Soft Comput. J., vol. 41, pp. 428-452, 2016, doi: 10.1016/j.asoc.2015.12.030.

[36] F. Jin, Z. Ni, H. Chen, Y. Li, and L. Zhou, "Multiple attribute group decision making based on interval-valued hesitant fuzzy information measures," Comput. Ind. Eng., vol. 101, pp. 103-115, 2016, doi: 10.1016/j.cie.2016.08.019.

[37] Y. Xu, F. J. Cabrerizo, and E. Herrera-Viedma, “A consensus model for hesitant fuzzy preference relations and its application in water allocation management," Appl. Soft Comput. J., vol. 58, pp. 265-284, 2017, doi:10.1016/j.asoc .2017.04.068

[38] S. Faizi, W. Sałabun, T. Rashid, J. Wątróbski, and S. Zafar, "Group Decision-Making for Hesitant Fuzzy Sets Based on Characteristic Objects Method," Symmetry (Basel)., vol. 9, no. 8, p. 136, 2017, doi: 10.3390/sym9080136.

[39] J. H. Lv, S. C. Guo, and F. F. Guo, "Study on Hesitant Fuzzy Information Measures and Their Clustering Application," Comput. Intell. Neurosci., vol. 2019, 2019, doi: 10.1155/20 19/5370763.

[40] Z. Xu and S. Zhang, "An overview on the applications of the hesitant fuzzy sets in group decision-making: Theory, support and methods," Front. Eng. Manag., vol. 6, no. 2, pp. 163-182, 2019, doi: 10.1007/s42524-019-0017-4.

[41] K. V Babitha and S. J. John, "Hesitant fuzzy soft sets," J. New Results Sci., vol. 3, pp. 98-107, 2013.
[42] F. Wang, X. Li, and X. Chen, "Hesitant Fuzzy Soft Set and Its Applications in Multicriteria Decision Making," J. Appl. Math., vol. 2014, pp. 1-10, 2014, doi:10.1155/2014/64378 5.

[43] I. Beg and T. Rashid, "Ideal solutions for hesitant fuzzy soft sets," J. Intell. Fuzzy Syst., vol. 30, no. 1, pp. 143-150, 2016, doi: $10.3233 /$ IFS-151740.

[44] K. Rezaei and H. Rezaei, "New distance and similarity measures for hesitant fuzzy soft sets," Iran. J. Fuzzy Syst., vol. 16, no. 6, pp. 159-176, 2019.

[45] C. Li, D. Li, and J. Jin, "Generalized Hesitant Fuzzy Soft Sets and Its Application to Decision Making," Int. J. Pattern Recognit. Artif. Intell., vol. 33, no. 12, pp. 1-30, 2019, doi: 10.1142/S0218001419500198.

[46] N. Çağman, F. Çıtak, and S. Enginoğlu, "Fuzzy Parameterized Fuzzy Soft Set Theory and Its Applications," Turkish J. Fuzzy Syst., vol. 1, no. 1, pp. 21-35, 2010.

[47] S. Alkhazaleh, A. R. Salleh, and N. Hassan, "Fuzzy Parameterized Interval-Valued," Appl. Math. Sci., vol. 5, no. 67, pp. 3335-3346, 2011.

[48] M. Bashir and A. R. Salleh, "Fuzzy Parameterized Soft Expert Set," Abstr. Appl. Anal., vol. 2012, pp. 1-15, 2012, doi: $10.1155 / 2012 / 258361$

[49] B. K. Tripathy and A. Panigrahi, "Interval-valued intuitionistic fuzzy parameterized soft set theory and its application in decision-making," Proc. 10th Int. Conf. Intell. Syst. Control. ISCO 2016, no. Iv, 2016, doi: 10.1109/ISCO .2016 .7726952 .

[50] S. Ganeshsree and S. Abdul Razak, "Fuzzy Parameterized Intuitionistic Fuzzy Soft Expert Set Theory and its Application in Decision Making," Int. J. Soft Comput., vol. 11 , no. 2, pp. 52-63, 2016, doi: 10.1504/ijads.2016.100009 83

[51] Y. Al-Qudah, M. Hassan, and N. Hassan, "Fuzzy Parameterized Complex Multi-Fuzzy Soft Expert Set Theory and Its Application in Decision-Making," Symmetry (Basel)., vol. 11, no. 3, p. 358, 2019, doi: 10.3390/sym110 30358.

[52] A. Al-Quran, N. Hassan, and S. Alkhazaleh, "Fuzzy Parameterized Complex Neutrosophic Soft Expert Set for Decision under Uncertainty," Symmetry (Basel)., vol. 11, no. 3, p. 382, 2019, doi: 10.3390/sym11030382.

[53] Z. Rodzi and A. G. Ahmad, "Fuzzy Parameterized Dual Hesitant Fuzzy Soft Sets and Its Application in TOPSIS," Math. Stat. 8, vol. 8, no. 1, pp. 32-41, 2020, doi: 10.13189 /ms.2020.080104.

[54] Z. Rodzi and A. G. Ahmad, "Fuzzy Parameterized Hesitant Fuzzy Linguistic Term Soft Sets (FPHFLTSSs) in Multi-Criteria Decision Making," Int. J. Innov. Technol. Explor.

Eng., vol. 9, no. 5, pp. 909-916, 2020, doi:10.35940/ijitee. E2519.039520.

[55] Z. Xu and M. Xia, "Distance and similarity measures for hesitant fuzzy sets," Inf. Sci. (Ny)., vol. 181, no. 11, pp. 2128-2138, 2011, doi: 10.1016/j.ins.2011.01.028.

[56] M. Xia and Z. Xu, "Hesitant fuzzy information aggregation in decision making," Int. J. Approx. Reason., vol. 52, no. 3, 
pp. 395-407, 2011, doi: 10.1016/j.ijar.2010.09.002.

[57] B. Farhadinia, "A series of score functions for hesitant fuzzy sets," Inf. Sci. (Ny)., vol. 277, pp. 102-110, 2014, doi: 10.1016/j.ins.2014.02.009.

[58] P. K. Maji, R. Biswas, and A. R. Roy, "Soft set theory," J. fuzzy Math., vol. 9, no. 3, pp. 589-602, 2003, doi: 10.1016/ S0898-1221(03)00016-6.

[59] D. Liu and L. Wang, "Multi-attribute decision making with hesitant fuzzy information based on least common multiple principle and reference ideal method," Comput. Ind. Eng., vol. 137, no. March, p. 106021, 2019, doi: 10.1016/j.cie.20 19.106021 .

[60] D. Li, W. Zeng, and J. Li, "New distance and similarity measures on hesitant fuzzy sets and their applications in multiple criteria decision making," Eng. Appl. Artif. Intell., vol. 40, pp. 11-16, 2015, doi: 10.1016/j.engappai.2014.12. 012. 